\title{
The ecological consequences and evolution of retron-mediated suicide as a way to protect bacteria from being killed by phage
}

\author{
Brandon A. Berryhill ${ }^{1,2,3}$, Rodrigo Garcia ${ }^{1,3}$, Joshua A. Manuel ${ }^{1}$, Bruce R. Levin ${ }^{1 *}$ \\ ${ }^{1}$ Department of Biology, Emory University \\ 1510 Clifton Rd NE, Atlanta, GA, 30322, USA \\ ${ }^{2}$ Program in Microbiology and Molecular Genetics (MMG), \\ Graduate Division of Biological and Biomedical Sciences (GDBBS), \\ Laney Graduate School, Emory University, Atlanta, GA, 30322, USA
}

${ }^{3}$ These authors contributed equally to this research

*Corresponding Author: Bruce R. Levin, Emory University, 1510 Clifton Rd Atlanta, GA 30322, 404-727-2826, blevin@emory.edu.

Author Contributions: BAB, RG, and BRL contributed to the experimental design, modelling, and analysis of the experiments as well as the writing of this report. JAM contributed to the execution of the experiments and the analysis of their results.

Competing Interests Statement: The authors have no competition interests to declare.

Keywords: Phage, Retron, Abortive infection, Population dynamics, Altruistic suicide, Bacterial defense systems 


\section{Abstract}

2 For more than thirty-five years since their discovery, the function of retrons, DNA sequences that 3 code for a reverse transcriptase and a unique single-stranded DNA/RNA hybrid called multicopy 4 single-stranded DNA (msDNA), in bacteria were unknown. Less than two years ago, compelling 5 evidence was presented that retrons can protect bacteria from infections from lytic phage via an 6 abortive infection mechanism. When infected with the virulent mutant of the phage lambda, $\lambda^{\text {VIR }}$, 7 and to a lesser extent other lytic phages, the retron designated Ec48 is activated, the Escherichia 8 coli bearing this element die, and the infecting phage is lost. Using mathematical models and 9 experiments with E. coli and $\lambda^{\mathrm{VIR}}$ we tested the hypothesis that Ec48 as an abortive infection system protects populations of bacteria from infection with lytic phage and we also tested the

11 hypothesis that phage-mediated selection is responsible for the evolution and maintenance of retrons. The results of this jointly theoretical and experimental investigation question this ecological role of retrons, and lytic phage as the selective pressure responsible for the evolution and maintenance of retrons in bacterial populations. We found that when confronted with phage, the retrons fail to provide an advantage to bacteria in mixed (retron ${ }^{+}$and retron $^{-}$) populations. Ultimately, bacteria with classical phage resistance are selected over bacteria utilizing retronmediated abortive infection.

\section{Significance Statement}

For thirty-six years, retrons have been known in bacteria. There have been numerous studies of the molecular biology and distribution of retrons. Recently, retrons have been used for genome editing, and are a tool considered to rival CRISPR-Cas for this purpose. However, only two years ago a function was identified for retrons: protection against infections with lytic bacteriophage. With a combination of mathematical modeling and experiments with retron-encoding $E$. coli we explore the ability of the retrons to protect bacterial populations from bacteriophage and the selective pressures responsible for their evolution. The results of this jointly theoretical and experimental study question whether protection against phage is the selective force responsible for the evolution and maintenance of retrons in bacterial populations. 


\section{Introduction}

Retrons, DNA sequences that code for a reverse transcriptase and a unique single-stranded DNA/RNA hybrid called multicopy single-stranded DNA (msDNA), were first discovered in 1984 (1) and were the first example of a reverse transcriptase coded for by bacteria $(2,3)$. Retrons were initially found in Myxococcus xanthus, but subsequently have been found in a number of bacterial species, including Escherichia coli $(4,5)$. Like CRISPR-Cas, retrons are being employed for genome engineering (5-7), and are capable of doing editing tasks that cannot be done by CRISPRCas (8). As was the case with CRISPR-Cas (9), for most of the time since its discovery the function of retrons was not known. That changed less than two years ago. Millman and collaborators presented compelling evidence that retrons in E. coli can defend against infections with lytic phages (10).

The mechanism by which retrons protect bacteria from lytic phages is known as abortive infection (abi), in which the infected cell dies and the infecting phage is lost (11). From an evolutionary perspective, abi is intriguing. With other anti-phage defenses, like envelope resistance, restrictionmodification, and most CRISPR-Cas systems in the presence of phage, these defense mechanisms are to the advantage of the individual bacteria expressing them. That is not the case for abortive infection. It has been postulated that abi is an altruistic character which provides an advantage to the population of clonal bacteria coding for these defense genes $(12,13)$. Individual cells that commit suicide due to phage infection, prevent the phage from infecting and killing other members of the population.

Previous work on abortive infection systems have shown the conditions under which abi systems have an advantage, and therefore, have provided a framework for testing other potential abortive systems. Fukuyo and colleagues as well as Berngruber and colleagues $(12,13)$, showed the protective role of abortive-infection mechanisms including specific conditions such as physicallystructured environments. Consequently, our Ec48 retron, which activates an abortive infection system, should be able to protect in similar conditions, however, both of these studies neglected to consider the contribution of classical resistance in their models and experiments with abortive phage infection.

Using a mathematical model of the population and evolutionary dynamics of bacteria and phage in mass culture, we explore the capacity of retron-mediated abortive infection to protect populations of bacteria from infection by lytic phages. We further explore the ability of phagemediated selection to favor retron-mediated immunity over envelope resistance. Using the retronencoding E. coli employed by Millman and colleagues (10) and a virulent mutant of the phage lambda, $\lambda^{\mathrm{VIR}}$, in liquid and structured (soft agar) cultures, we test the hypotheses generated from our theoretical analysis and those of Fukuyo and colleagues (12) and Berngruber and colleagues (13). The results of our experiments support the proposition that both in liquid and physically structured communities, retrons can protect populations of bacteria from infection by phage, but only when there are no other phage-sensitive bacterial populations supporting the replication of these bacterial viruses. Our results imply, consistent with the predictions of our models, that our abi system must be nearly $100 \%$ effective. These experiments also confirm the prediction that in mass, liquid culture with phage, retrons cannot evolve (increase when rare) in communities of bacteria without this defense system. Contrary to the prediction and experimental observations of Fukuyo and colleagues (12) our soft agar experiments suggest that even in this physically structured habitat, in the presence of phage, retrons cannot evolve solely by triggering abortive infection. The results of our experiments also provide evidence that the retron-mediated abi is only 
74 one element of this protection and invasion process. Within short-order of the bacterial populations 75 confronting phage in liquid and soft agar culture, $\lambda^{\mathrm{VIR}}$ resistant retron ${ }^{+}$and retron ${ }^{-}$bacteria emerge $^{-}$ 76 and become the dominant populations of bacteria.

77

\section{Results}

79 A model of retron-mediated abortive infection

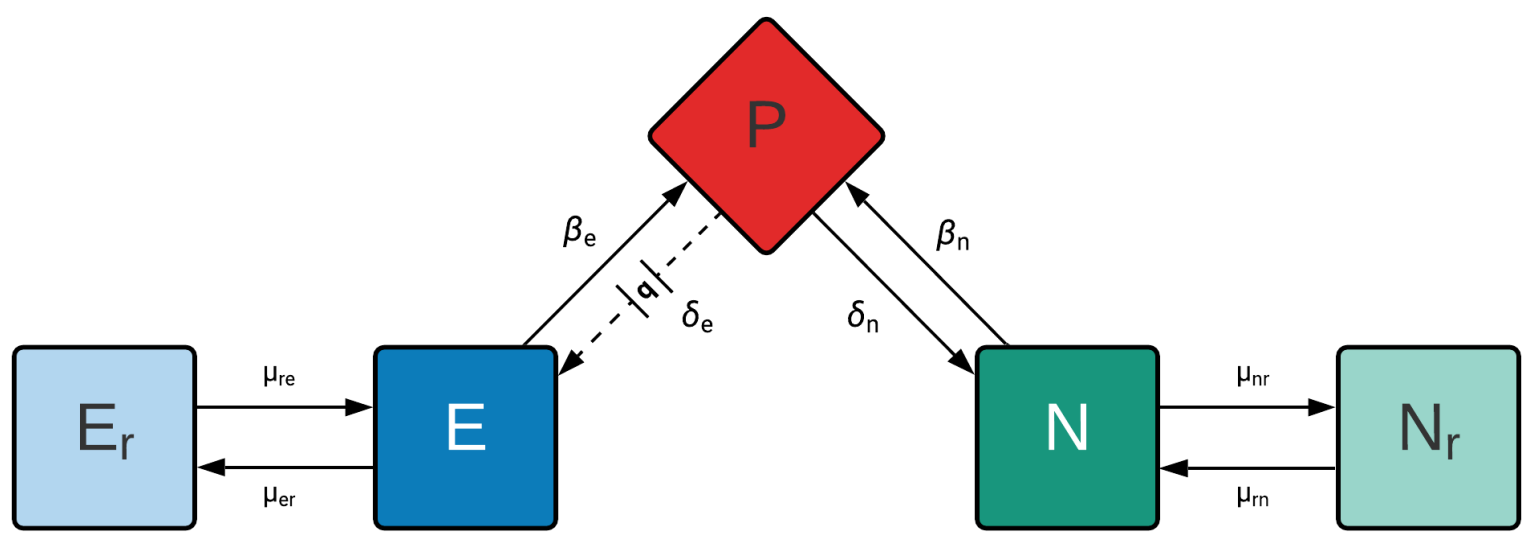

Figure 1. Model of the population and evolutionary dynamics of lytic phage with retron-mediated abortive infection. There are five populations: a lytic phage, $\mathrm{P}$; a phage-sensitive retron-encoding $\left(\right.$ retron $^{+}$) population, E; a phage-resistant retron ${ }^{+}$population, $\mathrm{E}_{\mathrm{r}}$; a phage-sensitive (retron ${ }^{-}$) population, $\mathrm{N}$; and a phage-resistant (retron ${ }^{-}$) population, $\mathrm{N}_{\mathrm{r}}$. The phage can adsorb at rates $\delta_{\mathrm{n}}$ and $\delta_{\mathrm{e}}$ to the $\mathrm{N}$ and $\mathrm{E}$ populations and replicate on $\mathrm{N}$ but only on a fraction (1-q) of the E population, bursting $\beta_{\mathrm{n}}$ and $\beta_{\mathrm{e}}$ phages particles, respectively. Bacteria can transition to $\left(\mu_{\mathrm{nr}}, \mu_{\mathrm{er}}\right)$ and from $\left(\mu_{\mathrm{rn}}\right.$, $\left.\mu_{\mathrm{re}}\right)$ their resistant and phage-sensitive states.

To build the theoretical background to generate our hypotheses, design our experiments, and to interpret their results, we generated a mathematical model. This model is based on the interactions of the populations illustrated in Figure 1, along with the definitions and assumptions detailed in the methods section. In accord with this model, the rates of change in the densities of bacteria and phage and the concentration of the limiting resource are given by the system of time-dependent, 


$$
\Psi(R)=\frac{R}{R+K}
$$

$$
\frac{d R}{d t}=-\Psi(R) \cdot e \cdot\left(v_{n} \cdot N+v_{n r} \cdot N_{r}+v_{e} \cdot E+v_{e r} \cdot E_{r}\right)
$$

$$
\frac{d N}{d t}=\Psi(R) \cdot\left(v_{n} \cdot N-\delta_{n} \cdot N \cdot P+\left(\mu_{r n} \cdot N_{r}-\mu_{n r} \cdot N\right)\right)
$$

$$
\frac{d N_{r}}{d t}=\Psi(R) \cdot\left(v_{n r} \cdot N_{r}-\left(\mu_{r n} \cdot N_{r}-\mu_{n r} \cdot N\right)\right)
$$

$$
\frac{d E}{d t}=\Psi(R) \cdot\left(v_{e} \cdot E-\left(\mu_{r e} \cdot E_{r}-\mu_{e r} \cdot E\right)\right)
$$

$$
\frac{d E_{r}}{d t}=\Psi(R) \cdot\left(v_{e r} \cdot E_{r}-\left(\mu_{r e} \cdot E_{r}-\mu_{e r} \cdot E\right)\right)
$$

$$
\frac{d P}{d t}=\Psi(R) \cdot\left(\delta_{n} \cdot \beta_{n} \cdot P \cdot N \cdot-\delta_{e} \cdot P \cdot q \cdot E+(1-q) \cdot \delta_{e} \cdot P \cdot E \cdot \beta_{e}\right)
$$
infection

We open this consideration of the population and evolutionary dynamics of retron-mediated abortive phage infection with an analysis of the ability of retrons to provide populations protection from predation by lytic phage. For this theoretical and the subsequent experimental considerations of retron-mediated abi, we present the predicted and observed densities of bacteria and phage when their populations first encounter each other, time 0, and at 24 hours (Figure 2). The values of the parameters used in these simulations are listed in the legends to this figure and Table S1. We performed an analysis to determine the minimum efficacy of retron-mediated abortive infection needed to protect populations from phage infection based on a range of values of abi effectiveness (q of 0.1 to 1.0 , with steps of 0.01 ). We found that at least $98 \%$ of the population had to abort the 
121 infection to protect the population from phage. In other words, q must be at least 0.98 for retron122 mediated abi to be protective. With these results in mind, we selected a q value of 1.00 and 0.95 , 123 to illustrate abortive infection success and failure, respectively figures $2 \mathrm{~A}$ and $2 \mathrm{~B}$.
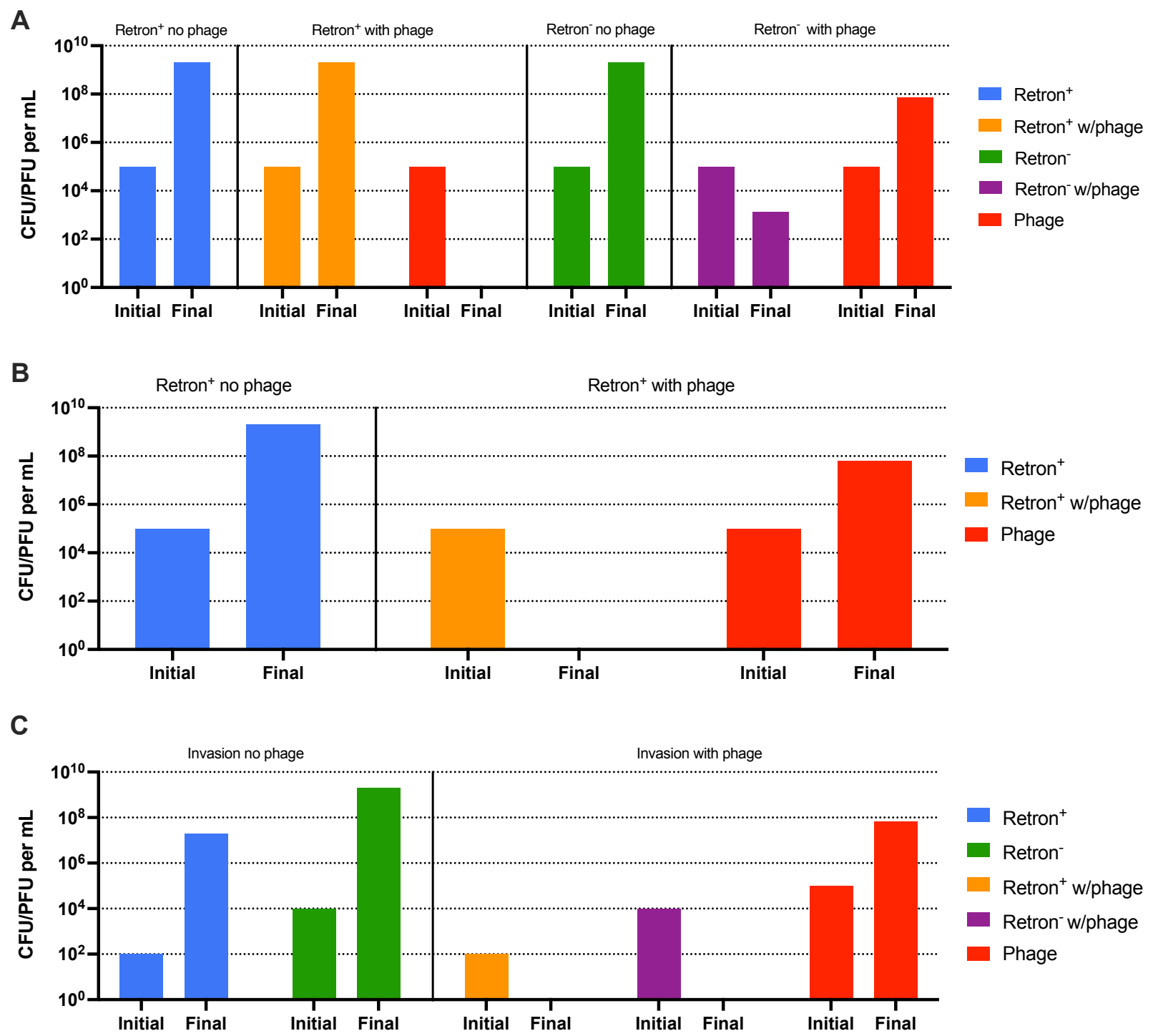

Figure 2. Computer simulation results without resistance. Changes in the densities of a retron ${ }^{+}$ bacterial population in the absence (blue) and presence (orange) of phage (red) and a retron ${ }^{-}$ bacterial population in the absence (green) and presence (purple) of phage at 0 (Initial) and 24 hours (Final). Standard parameters, $\mathrm{k}=1, \mathrm{e}=5 \times 10^{-7} \mu \mathrm{g} / \mathrm{cell}, \mathrm{v}_{\mathrm{e}}=\mathrm{v}_{\mathrm{n}}=2.0 \mathrm{~h}^{-1}, \delta_{\mathrm{e}}=\delta_{\mathrm{n}}=2 \times 10^{-7} \mathrm{~h}^{-1} \mathrm{cell}^{-}$ 1, $\beta_{\mathrm{a}}=\beta_{\mathrm{n}}=60$ phages $/$ cell, $\mu_{\mathrm{nr}}=\mu_{\mathrm{rn}}=\mu_{\mathrm{er}}=\mu_{\mathrm{re}}=0$. A- A completely effective $(\mathrm{q}=1.00)$ retron positive 130 (retron $\left.^{+}\right)$bacterial population in the absence and presence of phage. B- An incompletely effective $(\mathrm{q}=0.95)$ retron $^{+}$in the absence and presence of phage. $\mathbf{C}$ - Invasion of a completely effective $(\mathrm{q}=1.00)$ retron $^{+}$population into a high density of retron ${ }^{-}$bacteria in the absence and presence of phage. 
As can be seen in Figure 2A, a completely effective retron-mediated abi defense system ( $\mathrm{q}=1.00)$ is able to protect a population of retron ${ }^{+}$bacteria from predation by phage. By 24 hours, the phage population is gone and the retron ${ }^{+}$population is at its maximum density. When the retron ${ }^{-}$are confronted with phage, by 24 hours the bacteria are present at a low density, but there is a substantial density of free phage. The ability of the retron to prevent the ascent of the phage and protect the bacterial population is critically dependent on the efficacy of retron-mediated abortive infection (Figure 2A, 2B). When a mixed initial population of the retron ${ }^{+}$and retron ${ }^{-}$bacteria are $^{-}$ confronted with phage, the phage replicate on the retron ${ }^{-}$cells, the phage population increases in density and the retron ${ }^{+}$bacteria not only doesn't become established but is lost entirely (Figure 2C).

In Figure 3, we present the results of our experimental tests of the retron protection hypotheses presented in Figure 2 using the retron ${ }^{+} E$. coli Ec48 (10) and a lytic mutant of the phage lambda, $\lambda^{\mathrm{VIR}}$. As a retron ${ }^{-}$control, we use a $\lambda^{\mathrm{VIR}}$ sensitive $E$. coli $\mathrm{C}$. As anticipated from the model (Figure $2 \mathrm{~A}$ ), when confronting the retron ${ }^{+}$population by 24 hours, the $\lambda^{\mathrm{VIR}}$ population is gone or nearly so, and the bacterial density is at the level of a phage-free control (Figure 3A). The results of the control experiment with the $\lambda^{\text {VIR }}$ and the retron ${ }^{-}$sensitive strain are inconsistent with the prediction of the model (Figure 2B). As anticipated by the model, the phage density increased over the 24 hours, but contrary to what is expected from the model (Figure 2A), the bacteria are not lost, but 154 rather increased to the density anticipated in the absence of phage (Figure 3B). Also contrary to the prediction of the model (Figure 2C), in the presence of phage, the retron ${ }^{+}$population does not become extinct when initially rare relative to the retron ${ }^{-}$cells line. One possible reason for this, is that the bacteria recovered at 24 hours from the retron ${ }^{-}$population are resistant to $\lambda^{\mathrm{VIR}}$. To test this 158 hypothesis, we employed the cross-streak method on colonies isolated at 24 hours to determine their susceptibility to $\lambda^{\mathrm{VIR}}$ (Table S3). By this criterion, the vast majority of the initially sensitive 
A

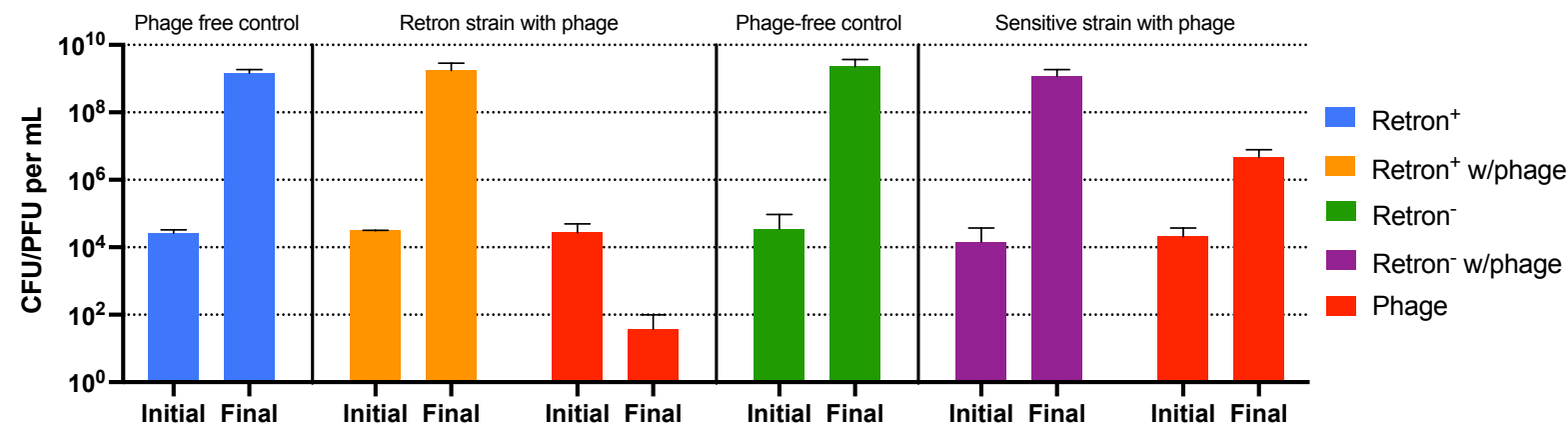

B

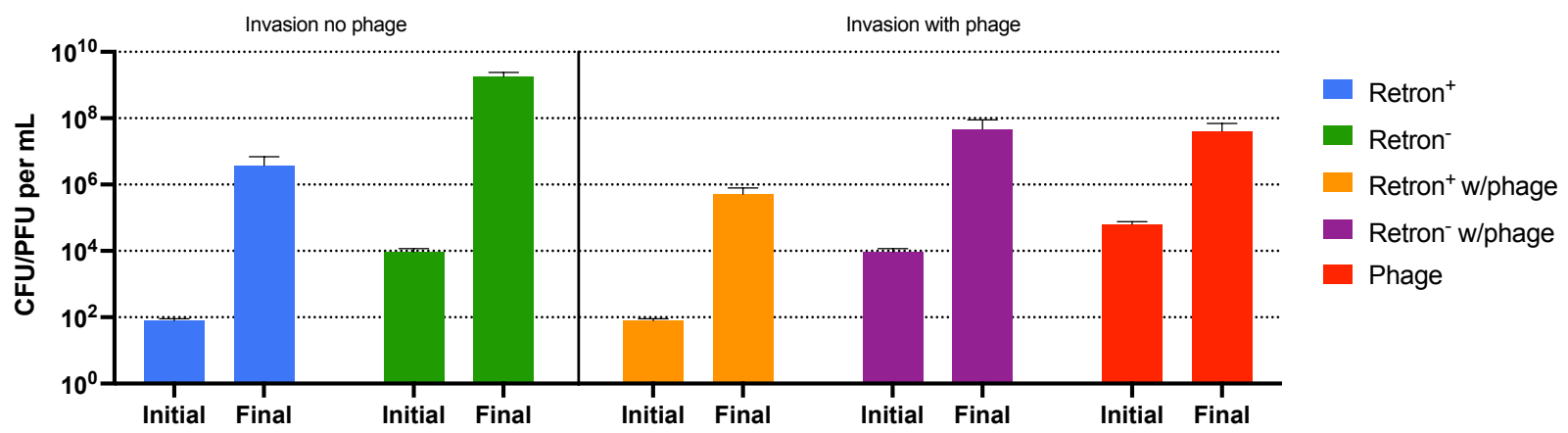

Figure 3. Experimental results, changes in the densities of bacteria and phage at 0 (Initial) and 24 (Final) hours. Retron ${ }^{+}$bacteria in the absence of phage (blue) and in the presence of $\lambda^{\text {VIR }}$ (orange), a retron- population of bacteria in the absence (green) of phage (red) and in the presence (purple) of phage. The data are the means and standard errors of the phage and bacterial densities of three independent replicas. A- Retron ${ }^{+}$and a retron ${ }^{-}$populations in the absence and presence of the lytic phage $\lambda^{\mathrm{VIR}}$. B- Invasion experiments. A co-culture of a low density of retron $^{+}$bacteria and a high density of retron ${ }^{-}$bacteria in the absence and presence of $\lambda^{\mathrm{VIR}}$.

To elucidate the reasons for the deviation from the predictions of the model, we performed new computer simulations with our model, but now allowed for the generation of a $\lambda^{\mathrm{VIR}}$ resistant retron ${ }^{+}$ population $\left(E_{r}\right)$ and a $\lambda^{\text {VIR }}$ resistant retron- $\left(N_{r}\right)$ bacterial population (Figure 1). As noted in Chaudhry et al (14), there is a high rate of generation of $\lambda^{\mathrm{VIR}}$ resistant bacteria for E. coli, suggesting transition rates, $\mu_{\mathrm{er}}$ and $\mu_{\mathrm{re}}$, of $10^{-5}$ per cell per hour. If we allow for that high transition rate for sensitive retron ${ }^{-}$cells, $\mu_{\mathrm{nr}}$ and $\mu_{\mathrm{rn}}$ of $10^{-5}$ per cell per hour, both the phage and resistant bacteria ascend (Figure 4). This parallels our observation in the previous experiments with $\lambda^{\text {VIR }}$ and a sensitive E. coli (Figure 3C). This is consistent with what we observed. If we allow for phage resistant mutants to be generated in our model, the retron ${ }^{+}$population can increase in density 
A

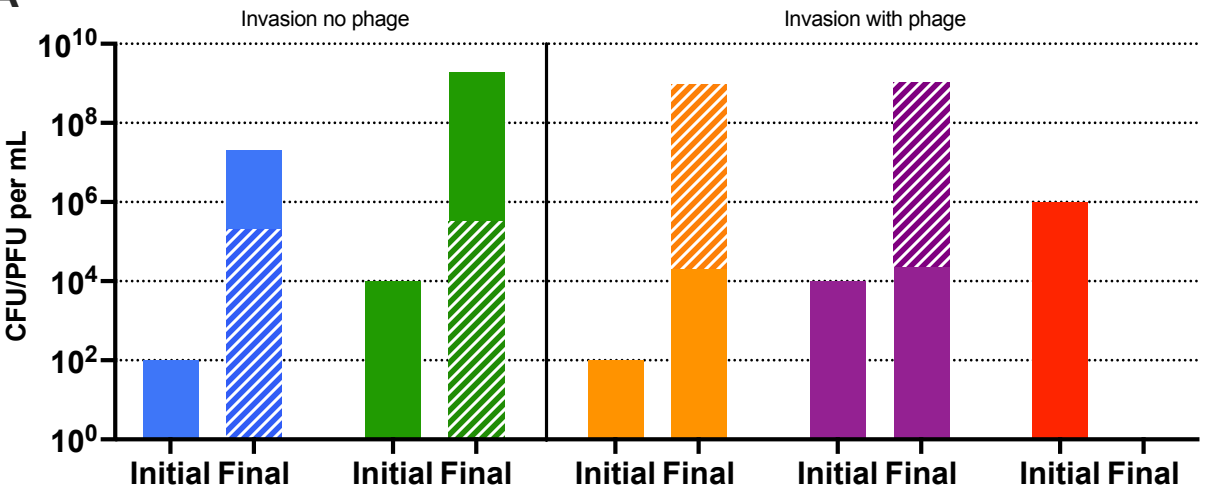

B
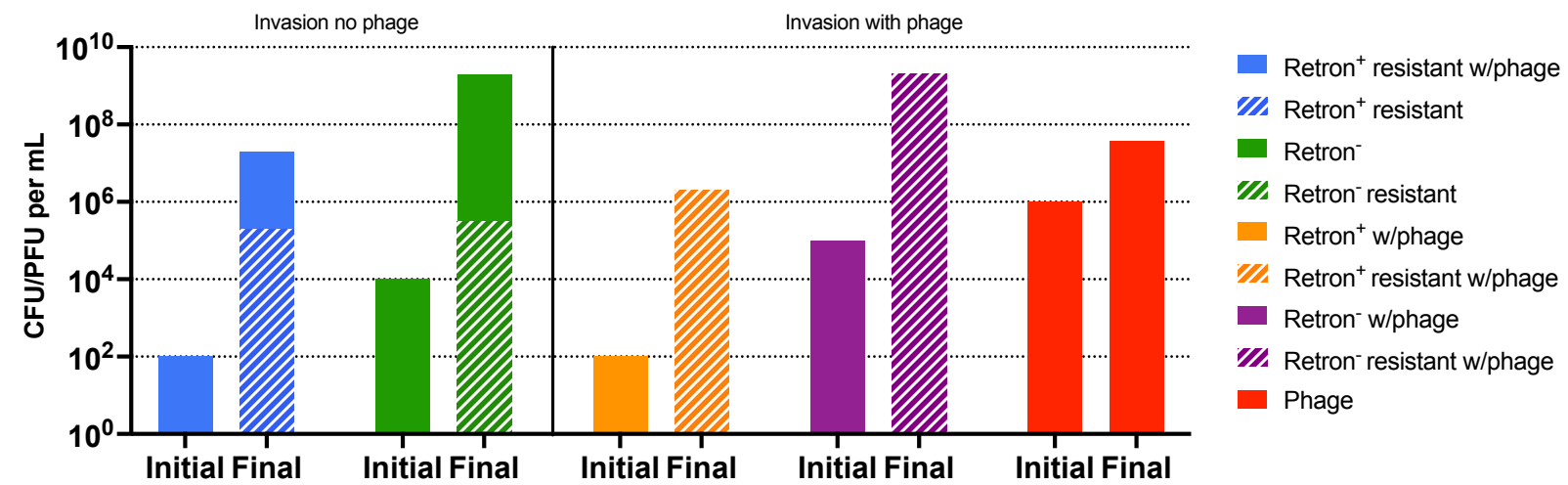

Figure 4. Computer simulations with resistance. The simulation conditions are similar to those in Figure $2 \mathrm{C}$ but allow for the generation and selection of resistance, $\mu_{\mathrm{er}}=\mu_{\mathrm{re}}=\mu_{\mathrm{nr}}=\mu_{\mathrm{rn}}=10^{-5}$ per cell per hour. Changes in the densities of bacteria and phage at time 0 (Initial) and 24 (Final) hours for two invasion conditions. Shown in the left side of this figure, are the densities of retron ${ }^{+}$(blue) and retron ${ }^{-}$(green) bacteria in the absence of phage. On the right side are the densities of retron ${ }^{+}$ (orange) and retron- bacteria (purple) in the presence of phage (red). The densities of phage resistant mutants are noted by bars with white hashing, while the sensitive populations are overlayed on the same bars. A-Simulations with a complete effective retron-mediated abi system $(\mathrm{q}=1.00)$. B- Simulations with a less-than completely effective retron-mediated abi system $(\mathrm{q}=0.95)$. 


\section{$\underline{\text { Retron-mediated abortive infection in a physically structured environment }}$}

202

203

204

205

206

207

208

209

210

211

212

213

214

215

216

217

218

219

220

221

222

223

224

225

226

227

228

229

230

231

232

233

234

With our demonstration of the failure of retrons to evolve in well-mixed culture, we performed parallel experiments in structured environments comparable to those employed by Fukuyo and colleagues (12). To explore the contribution of physical structure we performed the retron protection and the invasion when rare experiments depicted in Figure 3B in soft agar. As observed in liquid culture, in the physically structured habitat of soft agar, retron-mediated abi system protect the bacterial population from being killed off by the phage and prevents the replication of the phage (Figure 5). The retron-lacking population grows to maximum density and the phage are lost. As observed in liquid culture (Figure $3 \mathrm{~B}$ ), the retron ${ }^{+}$population is not favored by phagemediated selection. Also, as observed in liquid culture, all of the $\lambda^{\text {VIR }}$ exposed bacteria recovered at 24 hours were resistant to the phage (Table S3).

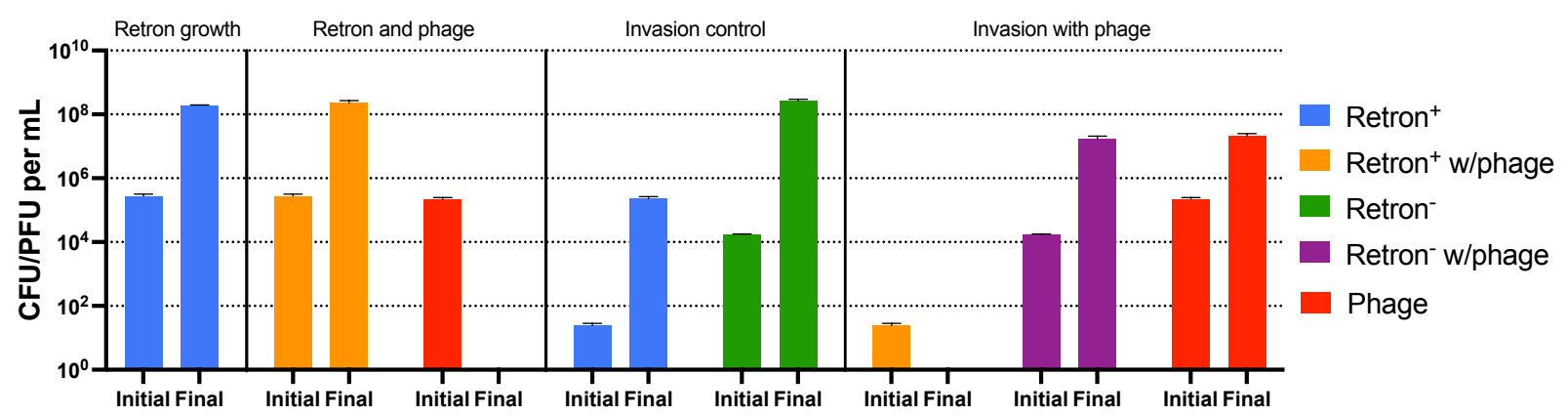

Figure 5. Experimental replicates of the results presented in Figure 3, but in a structured environment, presented as means and standard errors from three independent replicas. Shown are the densities of retron ${ }^{+}$(blue) and retron- (green) bacteria in the absence of phage, and the densities of retron $^{+}$(orange) and retron- bacteria (purple) in the presence of phage (red).

\section{Discussion}

Our mass action (liquid culture) models predict that a retron-mediated abortive infection system can prevent the populations of bacteria coding for them from being invaded by lytic phages, but only if the retron-mediated abi is nearly completely effective and when there are no other bacterial populations that can support the replication of the phage. The results of our experiments, like those of Millman and colleagues (10) with the retron-encoding abortive infection system in E. coli, Ec48, and the phage $\lambda^{\mathrm{VIR}}$, are consistent with these predictions. In addition to this protection result occurring in liquid culture, our results demonstrate that this protection against lytic phages happens in physically structured populations of bacteria maintained in soft agar. Our model also predicts if retron-mediated abortive infection is less than $98 \%$ effective, with more than $2 \%$ of infections being lytic and producing phage, or when there are retron ${ }^{-}$populations that can support the replication of the phage, retrons will not be able to protect a population from predation by lytic phages. We were unable to formally test this $<98 \%$ efficacy hypothesis experimentally, because our experimental results show that the retron-mediated abortive infection is overshadowed by selection for resistance to the phage. However, since our retron ${ }^{+}$population was capable of eliminating the phage population when alone, we interpret this to suggest that the efficacy of the Ec48 abortive infection system is over 98\%. Even though this retron-mediated abortive infection system is highly effective, when the bacteria are capable of generating envelope resistant mutants, 
in the presence of phage, retron $^{+}$or retron $^{-}$resistant mutants ascend to dominate the bacterial 236 populations.

237 Our model predicts that even if a retron-mediated abi defense system is $100 \%$ effective in preventing lytic phage replication, and there is an abundance of phage, the retron ${ }^{+}$population will not be able to evolve by abortive infection alone. Stated another way, retron ${ }^{+}$bacteria will not be able to become established in a population of retron ${ }^{-}$bacteria of similar fitness. Our experiments testing this hypothesis were consistent with this prediction. In liquid culture, the retron-expressing populations were unable to become established in populations dominated by retrons ${ }^{-}$competitors. In these experiments, the $E$. coli population surviving this encounter with $\lambda^{\mathrm{VIR}}$, was dominated by retron-lacking $\lambda^{\mathrm{VIR}}$ resistant mutants.

Our failure to see the ascent of retron ${ }^{+}$bacteria in soft agar is inconsistent with the prediction of the agent-based models Fukyou and colleagues (12) and Bergruber and colleagues (13) which demonstrated that in physically structured communities, there are conditions where, in the presence of phage, bacteria with abi systems can invade and become established in populations dominated by abi- cells sensitive to that phage. By adding $\lambda$ phage bearing a DNA methyltransferase Fukuyo and colleagues (12) constructed an E. coli with an abi system. Upon infections with a clear mutant of the phage lambda, $\lambda^{\mathrm{CL}}$ (which cannot form lysogens) cells with this abi construct die and the infecting phage is lost. In the physically structured habitat of soft agar $(12,15)$, in the presence $\lambda^{\mathrm{CL}}$ this abi-encoding $E$. coli has an advantage over $E$. coli without this abi system, but not in a habitat without this structure. Similar results were obtained by Berngruber and colleagues (13) with a lit mutant E. coli which, upon infection with the lytic phage T6, dies and aborts the infection. In their experiments with E. coli growing as colonies in structed environments, depending on the number and size of the colonies, bacteria with their lit abi system were substantially more fit than the competing population of abi $E$. coli. In neither of these studies, was the abortive infection able to evolve in liquid culture. Contrary to the results of Fukuyo and colleagues (12), with the retron-mediated abi $\lambda^{\text {VIR }}$ system used in this study, the retron-encoding population was unable to evolve in the physically structured habitat of soft agar.

We end on a somewhat philosophical note. People commonly assume that the phenotype observed is the object of natural selection. For example, resistance generated by modifications of the receptor sites to which phage adsorb evolves through selection mediated by phage. That selection can be easily demonstrated by exposing sensitive bacteria to phage. There is, however, another side to this. Clearly, the receptor sites to which the phage adsorb did not evolve to adsorb phage, no more than CRISPR and retrons evolved to enable molecular biologists to do genome editing. Throughout this investigation we, and almost all of the abortive infection articles cited, implicitly or explicitly assert that abi evolved in response to selection mediated by phage. Could it be that

272 retrons and other abi systems evolved and are maintained by selection for factors other than as defenses against phage infection? 
Materials and Methods

Mathematical modelling

In Figure 1, we illustrate our model of the population dynamics of lytic phage and bacteria with and without a retron-mediated abortive infection system and envelope resistance. There is a single population of phage, $\mathrm{P}$, particles per $\mathrm{ml}$ and four bacterial populations of bacteria, $\mathrm{E}, \mathrm{E}_{\mathrm{r}}, \mathrm{N}$, and $\mathrm{N}_{\mathrm{r}}$ cells per $\mathrm{ml}$. The phage sensitive retron population, $\mathrm{E}$, has a functional abi system. Though it also has a function abi system, the $E_{r}$ population is refractory to the phage. The $\mathrm{N}$ and $\mathrm{N}_{\mathrm{r}}$ populations are retron negative, retron', that are, respectively sensitive and resistant to the phage. When a phage infects a bacterium of state $\mathrm{E}$, there is a probability $\mathrm{q}(0 \leq \mathrm{q} \leq 1)$, that the bacteria will die and the infecting phage will be lost. The $\mathrm{N}$ population and 1-q of the E population support the replication of the phage while $E_{r}$ and $N_{r}$ are refractory to the phage. The bacteria grow at maximum rates, $\mathrm{v}_{\mathrm{e}}, \mathrm{ver}_{\mathrm{e}}, \mathrm{v}_{\mathrm{n}}$, and $\mathrm{v}_{\mathrm{nr}}$, per cell per hour, for $\mathrm{E}, \mathrm{E}_{\mathrm{r}}, \mathrm{N}$ and $\mathrm{N}_{\mathrm{r}}$, respectively with the net rate of growth being equal to the product of maximum growth rate, $V_{\max }$ and the concentration of a limiting resource, $\mathrm{r} \mu \mathrm{g} / \mathrm{ml}, \mathrm{v}_{\max } * \psi(\mathrm{R})(16), \mathrm{Eq}(1)$. The parameter $\mathrm{k}$, the Monod constant, is the concentration of the resource, at which the net growth rate of the bacteria is half its maximum value. By mutation or other processes, the bacteria change states, $\mathrm{E} \rightarrow \mathrm{E}_{\mathrm{r}}$ and $\mathrm{E}_{\mathrm{r}} \rightarrow \mathrm{E}$ at rates $\mu_{\mathrm{er}}$ and $\mu_{\mathrm{re}}$, per cell per hour, and $\mathrm{N} \rightarrow \mathrm{N}_{\mathrm{r}}$ and $\mathrm{N}_{\mathrm{r}} \rightarrow \mathrm{N}$ at rates $\mu_{\mathrm{nr}}$ and $\mu_{\mathrm{rn}}$.

The limiting resource is consumed at a rate equal to the product of $\psi(R)$, a conversion efficiency parameter, e $\mu \mathrm{g} /$ cell (17) and the sum of products of the maximum growth rates of the bacteria and their densities. We assume phage infection is a mass action process that occurs at a rate equal to the product of the density of bacteria and phage and a rate constants of phage infection, $\delta_{\mathrm{e}}$ and $\delta_{\mathrm{n}}$ (ml-cells/hour) for infections of $\mathrm{E}$ and $\mathrm{N}$, respectively (18). Infections of $\mathrm{N}$ by $\mathrm{P}$ produce $\beta_{\mathrm{n}}$ phage particles, and the (1-q) of the infections of $\mathrm{E}$ by $\mathrm{P}$ that do not abort, produce $\beta_{\mathrm{e}}$ phage particles. To account for the decline in physiological state as the bacteria approach stationary phase, $\mathrm{R}=0$, we assume phage infection and mutation rates decline at a rate proportional to Eq.1. The lag before the start of bacterial growth and latent period of phage infection are not considered in this model or the numerical solution employed to analyze its properties.

\section{Growth media and strains}

Bacterial cultures were grown at $37^{\circ} \mathrm{C}$ in MMB broth (LB broth (244620, Difco) supplemented with $0.1 \mathrm{mM} \mathrm{MnCl}_{2}$ and $5 \mathrm{mM} \mathrm{MgCl}_{2}$ ). The $E$. coli strain containing the Ec48 retron plasmid was obtained from Rotem Sorek. The sensitive $E$. coli used for controls was $E$. coli $\mathrm{C}$ marked with streptomycin resistance, and the Ec48 was marked with ampicillin resistance to differentiate in the invasion experiments. The $\lambda^{\mathrm{VIR}}$ phage lysates were prepared from single plaques at $37^{\circ} \mathrm{C}$ in LB medium alongside $E$. coli $\mathrm{C}$. Chloroform was added to the lysates and the lysates were centrifuged to remove any remaining bacterial cells and debris. The $\lambda^{\mathrm{VIR}}$ strain used in these experiments was obtained from Sylvain Moineau.

312 Bacteria and phage densities were estimated by serial dilution in $0.85 \%$ saline followed by 
experiments, diluted samples were placed on LB hard (1.6\%) agar plates supplemented with ampicillin $(2.5 \%)$ or streptomycin (4\%) plates to distinguish retron ${ }^{+}$and retron $E$. coli. To estimate the densities of free phage, chloroform was added to suspensions before serial dilution. These suspensions were plated at various dilutions on lawns made up of $0.1 \mathrm{~mL}$ of overnight LBgrown cultures of $E$. coli $\mathrm{C}$ (about $5 \times 10^{8}$ cells per $\mathrm{mL}$ ) and $4 \mathrm{~mL}$ of LB soft $(0.65 \%)$ agar on top of hard $(1.6 \%)$ LB agar plates.

\section{Resistance Testing with Cross Streaks}

Bacteria were tested by streaking in straight lines ten colonies from 24-hour plates across $20 \mu \mathrm{L}$ of a $\lambda^{\text {VIR }}$ lysate $\left(>10^{8}\right.$ plaque-forming units $\left.[\mathrm{pfu}] / \mathrm{mL}\right)$ on LB hard $(1.6 \%)$ agar plates. Susceptibility to $\lambda^{\mathrm{VIR}}$ was noted as breaks in the lines of growth. Continuous lines were

\section{Growth Rate Estimations}

Growth rates were estimated in a Bioscreen C. 48-hour overnights of each strain to be tested were diluted in MMB broth to an initial density of approximately $10^{5}$ cells per $\mathrm{ml}$. 10 replicas of each strain were loaded into 100 -well plates and grown at $37 \mathrm{c}$ with shaking for 24 hours taking OD $(600 \mathrm{~nm})$ measurements every five minutes.

\section{The Liquid culture experiments}

Bacterial overnight cultures grown at $37{ }^{\circ} \mathrm{C}$ in MMB Broth were serially diluted in $0.85 \%$ saline to approximate initial density and $100 \mu \mathrm{L}$ were added to flasks containing $10 \mathrm{~mL} \mathrm{MMB}$. $\lambda^{\mathrm{VIR}}$ lysate $\left(>10^{8} \mathrm{pfum} / \mathrm{ml}\right)$ was serially diluted to an MOI of $\sim 1$ and $100 \mu \mathrm{L}$ was added to the appropriate flask. These flasks were sampled for both phage and bacterial initial densities $(\mathrm{t}=0$ h) and were then grown at $37^{\circ} \mathrm{C}$ with constant shaking. The flasks were, once again, sampled for phage and bacterial densities $(\mathrm{t}=24 \mathrm{~h})$.

Bacterial cultures grown at $37^{\circ} \mathrm{C}$ in $\mathrm{MMB}$ and $\lambda^{\mathrm{VIR}}$ lysate were serially diluted in $0.85 \%$ saline to appropriate initial densities. The final dilutions were sampled for phage and bacterial initial densities and $100 \mu \mathrm{L}$ of diluted phage and bacteria were added to $4 \mathrm{~mL}$ of LB soft $(0.65 \%)$ agar and poured into small petri dishes which were grown at $37^{\circ} \mathrm{C}$. After 24 hours, the agar was placed into a tube containing $6 \mathrm{~mL}$ of saline, vortexed and sonicated in a water bath for 1 hour. These tubes were serially diluted and sampled for final phage and bacterial densities.

\section{Numerical solutions - computer simulations.}

345 To analyze the properties of this model we use Berkeley Madonna to solve the differential 346 Equations (1) - (7). The growth rate and phage infections parameters used for these simulations 347 are those estimated for $E$. coli and $\lambda^{\mathrm{VIR}}$. Copies of this program are available at www.eclf.net.

\section{Acknowledgements}

We thank Rotem Sorek and his colleagues at the Weitzmann Institute for providing us with the phage and bacteria used in this study. We are grateful to Ingrid McCall and David Goldberg, for advice and help with the experimental work. Funds for this research were provided a grant from the US National Institutes of General Medical Sciences, R35 GM 136407 (BRL). 


\section{References}

354 1. Yee T, Furuichi T, Inouye S, \& Inouye M (1984) Multicopy single-stranded DNA 355 isolated from a gram-negative bacterium, Myxococcus xanthus. Cell 38(1):203-209.

356 2. Inouye S, Hsu MY, Eagle S, \& Inouye M (1989) Reverse transcriptase associated with

3. Temin HM (1989) Reverse transcriptases. Retrons in bacteria. Nature 339(6222):254-

4. Simon DM \& Zimmerly S (2008) A diversity of uncharacterized reverse transcriptases in

5. Rice SA \& Lampson BC (1995) Phylogenetic comparison of retron elements among the

6. Rice SA, Bieber J, Chun JY, Stacey G, \& Lampson BC (1993) Diversity of retron elements in a population of rhizobia and other gram-negative bacteria. $J$ Bacteriol 175(13):4250-4254.

7. Simon AJ, Ellington AD, \& Finkelstein IJ (2019) Retrons and their applications in

8. Brownell L (2021) Move over CRISPR, the retrons are coming. in Wyss Institute Impact

9. Ishino Y, Shinagawa H, Makino K, Amemura M, \& Nakata A (1987) Nucleotide sequence of the iap gene, responsible for alkaline phosphatase isozyme conversion in Escherichia coli, and identification of the gene product. J Bacteriol 169(12):5429-5433.

10. Millman A, et al. (2020) Bacterial Retrons Function In Anti-Phage Defense. Cell

15. Chao L \& Levin BR (1981) Structured habitats and the evolution of anticompetitor toxins

16. Monod J (1949) The growth of bacterial cultures. Annual Review of Microbiology 3:371competition: a model and some general considerations. American Naturalist 107:171198. 
bioRxiv preprint doi: https://doi.org/10.1101/2021.05.05.442803; this version posted August 25, 2021. The copyright holder for this preprint (which was not certified by peer review) is the author/funder, who has granted bioRxiv a license to display the preprint in perpetuity. It is made available under aCC-BY-NC-ND 4.0 International license.

392 18. Chao L, Levin BR, \& Stewart FM (1977) A complex community in a simple habitat: an 393 experimental study with bacteria and phage. Ecology 58:369-378.

394 